\title{
Local limiting behavior of the zeros of approximating polynomials
}

\author{
M. Simkani \\ The University of Michigan-Flint, Department of Mathematics, Flint, MI 48502-2186, USA
}

\begin{abstract}
Let $f$ be a piecewise analytic (but not analytic) function in $C^{k}[a, b], k \geqslant 0$, and let $p_{n}^{*}$ be the sequence of polynomials of best uniform approximation to $f$ on $[a, b]$. It is well known that every point of $[a, b]$ is a limit point of the zeros of the $p_{n}^{*}$. Let $x \in[a, b]$, and suppose that $f$ is analytic at $x$ and $f(x) \neq 0$. The main purpose of this paper is to show that there exists a constant $\gamma$ (which depends only on $x$ ) such that there is no zero of $p_{n}^{*}$ within the circle of radius $(\gamma / n) \log n$ centered at $x$, for all sufficiently large values of $n$.
\end{abstract}

Key words: Piecewise analytic functions; Polynomials of best uniform approximation; Zeros; Points of singularity; Points of analyticity

\section{Introduction}

Let $E$ be a compact boundary set $(\operatorname{int}(E)=\emptyset$ ) in the complex plane whose complement is connected and regular in the sense that the complement has Green's function $G(z)$ with pole at $\infty$ (Walsh [8, p. 65]). Let $f$ be a continuous function on $E$, and for each positive integer $n$ let $p_{n}^{*}$ be the polynomial of degree at most $n$ of best uniform approximation to $f$ on $E$ :

$$
\left\|f-p_{n}^{*}\right\|_{E}<\|f-p\|_{E},
$$

for every polynomial $p \neq p_{n}^{*}$ of degree at most $n$. It is well known by Mergelyan's theorem [6, p. 423] that the left member of the above inequality tends to 0 as $n \rightarrow \infty$.

Now, consider a theorem of Blatt and Saff [2]:

Theorem 1.1. If $f$ has at least one point of singularity in $E$ (which means that if there is a point in $E$ at which $f$ is not analytic), then every point of $E$ is a limit point of the zeros of the $p_{n}^{*}$.

The converse of Theorem 1.1 is a consequence of the following well-known result:

Theorem 1.2. If there is a limit point $x \in E$ of the zeros of the $p_{n}^{*}$ and $f(x) \neq 0$, then $f$ has at least one point of singularity in $E$. 
The above theorems imply the following statement: When and only when there is a point of singularity (somewhere) in $E$, all points of $E$ (the points of analyticity as well as the points of singularity) attract zeros. This statement characterizes the existence of at least one point of singularity in terms of a global limiting behavior of the zeros.

In the present paper, we investigate the truth of the following hypothesis, which characterizes the points of singularity in terms of a local limiting behavior of the zeros:

Hypothesis. The points of singularity attract zeros faster than the points of analyticity.

The main results are stated in Section 2, and the proofs are given in Scction 3. We conclude this introduction with the following definitions:

Set

$$
E_{\rho}:=\{z: G(z)<\log \rho\},
$$

and $\Gamma_{\rho}:=\partial E_{\rho}$, for $\rho>1$. Any open neighborhood of $E$ contains some $E_{\rho}$, and according to Walsh [8, p. 65], $\Gamma_{\rho}$ "either consists of a finite number of finite mutually exterior analytic Jordan curves or consists of a finite number of contours which are mutually exterior except that each of a finite number of points may belong to several contours."

It is also known that if $f$ is analytic on $E_{\rho}$, then $p_{n}^{*}$ converges uniformly to $f$ on every compact subset of $E_{\rho}$ at a geometric rate. In fact, this can easily be used to prove Theorem 1.2.

For $E=[\alpha, \beta], \Gamma_{\rho}$ is the ellipse with foci $\alpha$ and $\beta$, whose major and minor semi-axes have lengths $\frac{1}{4}(\beta-\alpha)(\rho+1 / \rho)$ and $\frac{1}{4}(\beta-\alpha)(\rho-1 / \rho)$ respectively.

\section{Main results}

We begin this section with a theorem, which shows that the points of analyticity impose a certain speed limit on the approaching zeros:

Theorem 2.1. Let $f$ be a continuous (real or complex) function on $E$ that does not vanish at any point of $E$, and let $p_{n}$ be a sequence of polynomials (of respective degrees at most $n$ ) that converges to $f$ uniformly on $E$. Let $\rho_{n}$ be a decreasing sequence of real numbers approaching 1 , such that

$$
\rho_{n}^{n}\left\|f-p_{n}\right\|_{E} \rightarrow 0,
$$

as $n \rightarrow \infty$. If $f$ is analytic on $E$, then $p_{n}$ does not vanish at any point of $E_{\rho_{n}}$, for all sufficiently large values of $n$.

As a consequence of Theorem 2.1, consider a result on piecewise analytic functions:

Corollary 2.2. Let $t_{0}<t_{1}<\cdots<t_{m}$, and let $f$ be a function $k$ times continuously differentiable on $\left[t_{0}, t_{m}\right]$, such that $f$ is analytic on $\left(t_{i-1}, t_{i}\right)$ for every $i=1, \ldots, m$. Let $p_{n}^{*}$ be the sequence of polynomials (of respective degrees at most $n$ ) of best uniform approximation to $f$ on $\left[t_{0}, t_{m}\right]$. For some $j=1, \ldots, m$, let $x \in\left(t_{j-1}, t_{j}\right), c_{x}=\min \left\{x-t_{j-1}, t_{j}-x\right\}$, and $0<\gamma<c_{x}(k+1)$. Suppose that $f$ does not vanish in $\left(t_{j-1}, t_{j}\right)$, and let $\Delta_{n}(x)$ be the disk of radius $(\gamma / n) \log n$ centered at $x$. 
Then, $p_{n}^{*}$ does not vanish at any point of $\Delta_{n}(x)$ for all sufficiently large values of $n$. Furthermore, let $x=t_{j}$, for some $j=0, \ldots, m$, such that $f(x) \neq 0$. Suppose that either the restriction of $f$ on $\left(t_{j-1}, t_{j}\right]$ or the restriction of $f$ on $\left[t_{j}, t_{j+1}\right)$ is analytic at $t_{j}$, and let $c=\frac{1}{4}\left(t_{j}-t_{j-1}\right)$ or $\frac{1}{4}\left(t_{j+1}-t_{j}\right)$, respectively. For $0<\gamma<\sqrt{c}(k+1)$, let $\Delta_{n}(x)$ be the disk of radius $[(\gamma / n) \log n]^{2}$. Then, $p_{n}^{*}$ does not vanish at any point of $\Delta_{n}(x)$ for all sufficiently large values of $n$.

The following two conjectures are based on some preliminary numerical computations.

Conjecture 2.3. Let $f(x)=|x|($ or $f(x)=|x|+1)$, and let $p_{n}^{*}$ be the sequence of polynomials (of respective degrees at most $n$ ) of best uniform approximation to $f$ on $[-1,1]$. Then, there exists a constant $\delta<1.18$ ( or $<1.52$ ), such that for all sufficiently large values of $n, p_{n}^{*}$ vanishes in the disk of radius $(\delta / n) \log n$ centered at $x=\frac{1}{2}$.

Notice that for the functions of Conjecture 2.3, in Corollary 2.2, if $x=\frac{1}{2}$, then $\gamma<0.5$. This shows that the first part of Corollary 2.2 is sharp up to a constant.

The following conjecture supports our hypothesis that the points of singularity attract zeros faster than the points of analyticity.

Conjecture 2.4. Let $f(x)=|x|($ or $f(x)=|x|+1)$, and let $p_{n}^{*}$ be the sequence of polynomials (of respective degrees at most $n$ ) of best uniform approximation to $f$ on $[-1,1]$. Then there exists a constant $\kappa$, such that $p_{n}^{*}$ vanishes in $\Delta_{n}$ the disk of radius $\kappa / n$ centered at 0 , for all sufficiently large values of $n$.

How sharp is Theorem 2.1?

Theorem 2.5. Let $E=[-1,1]$, and let $\rho_{n}$ be a decreasing sequence of real numbers approaching 1 , such that

$$
\rho_{n}^{n}\left(\rho_{n}-1\right)^{2+\varepsilon} \rightarrow \infty
$$

for some $\varepsilon>0$. Then, for every function $f$ analytic on $E$, there exists a sequence $p_{n}$ of polynomials (of respective degrees at most $n$ ) that converges to $f$ uniformly on $E$, such that $p_{n}$ vanishes in $E_{\rho_{n}}$, for all sufficiently large values of $n$.

We conclude this section by referring the readers to $[1$, p. $196 ; 2-5 ; 9]$, for related results on the distribution of zeros.

\section{Proofs}

Proof of Theorem 2.1. The function $f$ is analytic and non-vanishing in $E_{\rho}$, for some $\rho>1$. For each positive integer $n$, let $p_{n}^{*}$ be the polynomial of degree at most $n$ of best uniform approximation to $f$ on $E$. Then

$$
\left|p_{n}^{*}(z)\right|>\varepsilon
$$

in $E_{\rho_{n}}$, for $n$ sufficiently large, and for some $\varepsilon>0$. 
By the Bernstein-Walsh Lemma [8, p. 77], for all $z \in E_{\rho_{n}}$, and for all sufficiently large values of $n$, we have

$$
\left|p_{n}(z)-p_{n}^{*}(z)\right| \leqslant \rho_{n}^{n}\left\|p_{n}-p_{n}^{*}\right\|_{E} \leqslant 2 \rho_{n}^{n}\left\|f-p_{n}\right\|_{E}<\frac{1}{2} \varepsilon,
$$

which yields

$$
\left|p_{n}(z)\right| \geqslant\left|p_{n}^{*}(z)\right|-\left|p_{n}(z)-p_{n}^{*}(z)\right|>\frac{1}{2} \varepsilon .
$$

This completes the proof.

Proof of Corollary 2.2. Let $t_{j-1}<x<t_{j}$, and $0<\gamma<c_{x}(k+1)$. Choose $\varepsilon>0$, such that

$$
0<\lambda:=\frac{2 \gamma}{\left(c_{x}-\varepsilon\right)(2-\varepsilon)}<k+1 .
$$

Then, let $E=\left[x-c_{x}+\varepsilon, x+c_{x}-\varepsilon\right]$, and

$$
\rho_{n}=1+\frac{\lambda \log n}{n} .
$$

It is easy to see that $\rho_{n}^{n}<n^{\lambda}$, and hence

$$
\rho_{n}^{n}\left\|f-p_{n}^{*}\right\|_{E} \rightarrow 0,
$$

since it is well known that $\left\|f-p_{n}^{*}\right\|_{E}<$ const $/ n^{k+1}$ [7, Chapter 7].

By Theorem 2.1, it remains to show that $\Delta_{n}(x) \subset E_{\rho_{n}}$. And for this, we may show that

$$
\frac{\gamma \log n}{n}<\frac{c_{x}-\varepsilon}{2}\left(\rho_{n}-\frac{1}{\rho_{n}}\right),
$$

by observing that

$$
\begin{aligned}
\rho_{n}-\frac{1}{\rho_{n}} & =\rho_{n}-1+\left(\rho_{n}-1\right)-\left(\rho_{n}-1\right)^{2}+\cdots \\
& >\left(\rho_{n}-1\right)\left(3-\rho_{n}\right)>\frac{\lambda \log n}{n}(2-\varepsilon),
\end{aligned}
$$

for $n$ large. This completes the first part of the proof.

Now suppose that $x=t_{j}$, for some $j=1, \ldots, m$ (the case where $j=0$ is similar), $f(x) \neq 0$, and the restriction of $f$ on $\left(t_{j-1}, t_{j}\right]$ is analytic at $t_{j}$ (the case where the restriction of $f$ on $\left[t_{j}, t_{j+1}\right)$ is analytic at $t_{j}$ is similar). Let $c=\frac{1}{4}\left(t_{j}-t_{j-1}\right), 0<\gamma<\sqrt{c}(k+1)$, and $\Delta_{n}(x)$ be the disk of radius $[(\gamma / n) \log n]^{2}$ centered at $x$.

Choose $\varepsilon>0$, such that

$$
\lambda:=\frac{\gamma}{\sqrt{(c-\varepsilon)(1-\varepsilon)}}<k+1 .
$$

Then, let $E=\left[t_{j-1}+4 \varepsilon, t_{j}\right]$, and $\rho_{n}=1+(\lambda / n) \log n$. It is sufficient to show that

$$
\left(\frac{\gamma \log n}{n}\right)^{2}<(c-\varepsilon)\left(\rho_{n}+\frac{1}{\rho_{n}}\right)-2(c-\varepsilon),
$$


by using

$$
\rho_{n}+\frac{1}{\rho_{n}}>2+\left(\rho_{n}-1\right)^{2}\left(2-\rho_{n}\right)>2+\left(\frac{\lambda \log n}{n}\right)^{2}(1-\varepsilon),
$$

for $n$ large. This completes the proof.

Proof of Theorem 2.5. Let

$$
\begin{array}{ll}
r_{n}=1+\frac{\rho_{n}-1}{1+\frac{1}{2} \varepsilon}, & b_{n}=\frac{1}{2}\left(r_{n}-r_{n}^{-1}\right), \quad \beta_{n}=\frac{1}{2}\left(\rho_{n}-\rho_{n}^{-1}\right), \\
y_{n}=\frac{1}{2}\left(b_{n}+\beta_{n}\right), & f_{n}(z)=\frac{f(z)}{z-\mathrm{i} y_{n}} .
\end{array}
$$

Each function $f_{n}$ is analytic on the closure of $E_{r_{n}}$, for all sufficiently large values of $n$, and i $y_{n} \in E_{\rho_{n}}$.

Let $p_{n-1}^{*}$ be the polynomial of degree at most $n-1$ of best uniform approximation to $f_{n}$ on $E$, for each $n$. Then, by Bernstein's error bound [1, p. 82], we have

$$
\left\|f_{n}-p_{n-1}^{*}\right\|_{E}<\frac{2 M_{n}}{r_{n}^{n-1}\left(r_{n}-1\right)}
$$

where

$$
M_{n}=\max _{z \in \Gamma_{r_{n}}}\left|f_{n}(z)\right| \leqslant \frac{M}{y_{n}-b_{n}} \leqslant \frac{K}{\varepsilon\left(r_{n}-1\right)},
$$

for some constants $M$ and $K$.

Condition (1) yields

$$
\rho_{n}^{n /(1+\varepsilon / 2)}\left(\rho_{n}-1\right)^{2} \rightarrow \infty
$$

which implies

$$
r_{n}^{n}\left(r_{n}-1\right)^{2} \rightarrow \infty
$$

and hence the left member of (2) tends to zcro.

Now, set

$$
p_{n}(z)=\left(z-\mathrm{i} y_{n}\right) p_{n-1}^{*}(z),
$$

which converges to $f$ uniformly on $E$, and this completes the proof.

\section{Acknowledgments}

The author thanks professors H. D'Souza, W. Pleśniak, E.B. Saff, A.P. Wójcik, and M. Wyneken for useful comments and discussions. The author is also indebted to the referee for the improvements in condition (1) and in the proof of Theorem 2.1. This work began while the 
author was enjoying the hospitality of the Jagiellonian University, Cracow, Poland. The research was supported (in part) by a grant from the Faculty Research Initiative Program of the University of Michigan-Flint.

\section{References}

[1] S.N. Bernstein, Collected Works, Vol. I: Constructive Theory of Functions (1905-1930) (Academy of Sciences USSR Press, Moscow, 1952).

[2] H.-P. Blatt and E.B. Saff, Behavior of zeros of polynomials of near best approximation, J. Approx. Theory 46 (1986) 323-344.

[3] H.-P. Blatt, E.B. Saff and M. Simkani, Jentzsch-Szegö type theorems for the zeros of best approximants, $J$. London Math. Soc. (2) 38 (1988) 307-316.

[4] P.B. Borwein, The relationship between the zeros of best approximation and differentiability, Proc. Amer. Math. Soc. 92 (1984) 528-532.

[5] W. Pleśniak, On the distribution of the zeros of the pulynomials of best $L^{2}$-approximation to holomorphic functions, Zeszyty Nauk. Uniw. Jagiell. Prace Mat. 22 (1981) 29-35.

[6] W. Rudin, Real and Complex Analysis (McGraw-Hill, New York, 2nd ed., 1974).

[7] A.F. Timan, Theory of Approximation of Functions of a Real Variable (Pergamon Press, New York, 1963).

[8] J.L. Walsh, Interpolation and Approximation by Rational Functions in the Complex Domain, AMS Colloquium Publications 20 (AMS, Providence, RI, 5th ed., 1969).

[9] A.P. Wójcik, On zeros of polynomials of best approximation to holomorphic and $C^{\infty}$ functions, Mh. Math. 105 (1988) $75-81$. 